\title{
Enantioselective reduction of prochiral aryl ketones using a novel chiral auxiliary derived from a labdane diterpene
}

\author{
Diego A. Cifuente* and Carlos E. Tonn \\ INTEQUI-CONICET, Facultad de Química, Bioquímica y Farmacia, Universidad Nacional de \\ San Luis, Chacabuco y Pedernera, 5700 San Luis, Argentina \\ E-mail: cifuente@unsl.edu.ar
}

Dedicated to Professor Oscar S. Giordano

\begin{abstract}
A novel chiral 2-methoxy-1,3,2-dioxaborolane derived from $2 \alpha, 3 \alpha$-dihydroxycativic acid, a labdane-type diterpene isolated from aerial parts of Baccharis scandens DC., is reported as a highly effective chiral auxiliary. This compound has been successfully used in the borane dimethyl sulfide complex-mediated asymmetric reduction of prochiral aryl ketones at room temperature. Very good chemical yields (up to 89\%) and high enantioselectivities were obtained.
\end{abstract}

Keywords: Asymmetric reductions, chiral 1,3,2-dioxaborolane, $2 \alpha, 3 \alpha$-dihydroxycativic acid, labdane-type diterpene

\section{Introduction}

Stereoselective reduction of prochiral ketones to their corresponding enantiopure secondary alcohols is a key step in the preparation of a variety of pharmaceutical products. ${ }^{1}$ Introduction of chirality in organic molecules through the use of chiral reagents is a valuable synthetic tool in organic chemistry. As such, the development of chiral auxiliaries or catalysts to perform reductions with high enantioselectivity has attracted much attention. New methods such as asymmetric transfer hydrogenation, ${ }^{2}$ metal catalyzed hydrogenations, ${ }^{3}$ enzymatic reactions ${ }^{4}$ and the use of metal hydride transfer reagents, ${ }^{5}$ have been reported.

The use of chiral boron reagents has gained importance during the last decade, ${ }^{6}$ and some oxazaborolidines function as excellent catalysts for enantioselective borane reduction of prochiral ketones. ${ }^{7}$ In addition, 1,3,2-dioxaborolanes and 1,3,2-dioxaborinanes are well known as chiral reagents in many regio-, stereo- and enantioselective reactions. ${ }^{8,9}$

On the other hand, numerous chiral auxiliaries and catalysts are derived from natural sources. Natural products isolated from plants are of particular interest because they can often mediate 
significant levels of stereoselection. In this way, boron reagents derived from chiral monoterpenes are useful in asymmetric synthesis; ${ }^{10}$ versatile pinane-based reagents have been successfully applied to many chiral transformations. ${ }^{11}$

In this paper we describe the synthesis and the chiral properties of a novel 2-methoxy-1,3,2dioxaborolane employed in borane-mediated asymmetric reduction of prochiral ketones. The 5membered cyclic chiral borate was prepared from $2 \alpha, 3 \alpha$-dihydroxycativic acid, a labdane-type diterpene isolated from aerial parts of Baccharis scandens DC. and trimethoxyborane. The borane-dimethylsufide complex was used as the hydride source. The corresponding optically active alcohols were achieved in high chemical yields (89-99\%) and good enantioselectivities $(64-98 \% e e)$. Additionally, the effect of chiral auxiliary-substrate ratio and temperature on the enantioselectivity is discussed.

\section{Results and Discussion}

From aerial parts of $B$. scandens DC., a species that grows in the semi-arid western region of Argentina, was isolated $2 \alpha, 3 \alpha$-dihydroxycativic acid (45 mg/500 g plant) (1). ${ }^{12}$ Methylation of the dihydroxycativic acid $\mathbf{1}$ with diazomethane followed by Pd-catalyzed hydrogenation yielded the methyl ester $\mathbf{2}$. The corresponding 2-methoxy-1,3,2-dioxaborolane $\mathbf{3}$ was synthesized in situ from the methyl ester 2 with trimethoxyborane in dry toluene at reflux for $8 \mathrm{~h}$ (Scheme 1). Its structure was supported by IR, ${ }^{1} \mathrm{H}$ and ${ }^{13} \mathrm{C}$ NMR spectral data. ${ }^{13,14}$

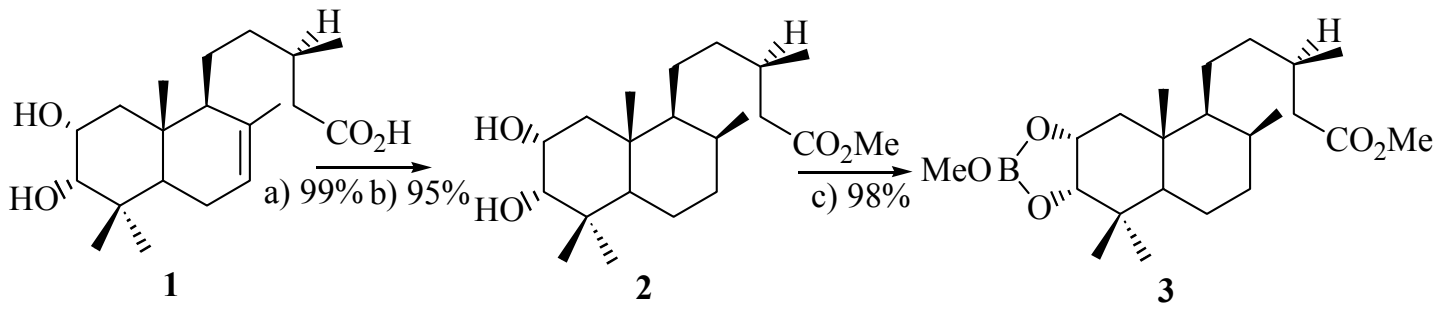

Scheme 1. Reagents and conditions: (a) $\mathrm{CH}_{2} \mathrm{~N}_{2}, 0{ }^{\circ} \mathrm{C}, \mathrm{Et}_{2} \mathrm{O}$; (b) $\mathrm{H}_{2}(\mathrm{Pd} / \mathrm{C}), \mathrm{MeOH}$; (c) $\mathrm{B}(\mathrm{OMe})_{3}$, PhMe, reflux, $8 \mathrm{~h}$.

In order to study the potential of this boronate ester 3 in the borane-mediated asymmetric reduction, we selected a series of alkylaryl ketones 4-10 as substrates. The dioxaborolane 3 (10 mol \%) was applied, without isolation, in the borane dimethylsulfide complex-mediated asymmetric reduction (Scheme 2).

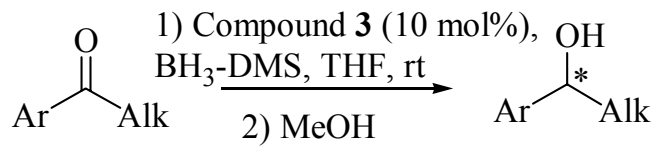

Scheme 2. Typical borane dimethylsulfide complex-asymmetric reduction of alkylaryl ketones. 
The corresponding optically active $R$-alcohols 11-17 were isolated in excellent chemical yields and good enantiopurity for sterically hindered ketones (Table 1).

Table 1. Results of enantioselective reduction of prochiral ketones 4-10 using 2-methyl-1,3,2dioxaborolane 3<smiles>[R]c1ccc(C(C)=O)cc1</smiles>

$4 \mathrm{R}=\mathrm{H} ; 5 \mathrm{R}=\mathrm{Cl} ; 6 \mathrm{R}=\mathrm{B}$

$7 \mathrm{R}=\mathrm{OMe} ; \mathbf{8} \mathrm{R}=\mathrm{Me}$<smiles>[R]c1ccc(C(C)O)cc1</smiles>

$11 \mathrm{R}=\mathrm{H} ; 12 \mathrm{R}=\mathrm{Cl} ; 13 \mathrm{R}=\mathrm{Br}$;

$14 \mathrm{R}=\mathrm{OMe} ; 15 \mathrm{R}=\mathrm{Me}$<smiles>O=C1CCc2ccccc21</smiles>

9<smiles>O[C@H]1CCc2ccccc21</smiles>

16<smiles>O=C1CCCc2ccccc21</smiles>

10<smiles>O[C@H]1CCCc2ccccc21</smiles>

17

\begin{tabular}{cccccc}
\hline Entry & Ketone & $e e(\%)$ & Conf. & Yield $^{\mathrm{e}}(\%)$ & Product \\
\hline 1 & $\mathbf{4}$ & $64^{\mathrm{a}}$ & $R^{\mathrm{d}}$ & 93 & $\mathbf{1 1}$ \\
2 & $\mathbf{5}$ & $91^{\mathrm{a}}$ & $R^{\mathrm{d}}$ & 89 & $\mathbf{1 2}$ \\
3 & $\mathbf{6}$ & $93^{\mathrm{a}}$ & $R^{\mathrm{d}}$ & 94 & $\mathbf{1 3}$ \\
4 & $\mathbf{7}$ & $89^{\mathrm{a}}$ & $R^{\mathrm{d}}$ & 91 & $\mathbf{1 4}$ \\
5 & $\mathbf{8}$ & $90^{\mathrm{a}}$ & $R^{\mathrm{d}}$ & 92 & $\mathbf{1 5}$ \\
6 & $\mathbf{9}$ & $95^{\mathrm{b}}$ & $R^{\mathrm{c}}$ & 98 & $\mathbf{1 6}$ \\
7 & $\mathbf{1 0}$ & $96^{\mathrm{b}}$ & $R^{\mathrm{c}}$ & 98 & $\mathbf{1 7}$ \\
\hline
\end{tabular}

${ }^{a}$ Enantiomeric excesses were determined by capillary chiral GC. ${ }^{b}$ Enantiomeric excesses were determinate by chiral HPLC (Daicel Chiralcel OD-H column). ${ }^{c}$ Absolute configurations determined by comparison of optical rotation with those described in the literature. ${ }^{\mathrm{d}}$ Absolute configurations determined by comparison of retention times by chiral GC analyses (Supelco $\beta$ DEX 120 column). ${ }^{\mathrm{e}}$ Chemical yields of isolated products.

We first carried out the asymmetric reduction of acetophenone 4 in the presence of $10 \mathrm{~mol} \%$ of chiral dioxaborolane $\mathbf{3}$ with borane dimethylsulfide in THF at room temperature. The reduction of 4 proceeded to give $(R)$-1-phenylethanol 11 in $93 \%$ yield and with $64 \%$ enantiomeric excess (entry 1$){ }^{15}$

Next, haloketones $\mathbf{5}$ and $\mathbf{6}$ derived from acetophenone were investigated under the same reaction conditions. Compounds 5 and $\mathbf{6}$ carrying, chlorine or bromine atoms at C-4 of the aryl group, gave the alcohols 12 and 13 ( $R$-form), respectively with moderate to good enantioselectivities (up to 91\%) and good chemicals yields (up to 89\%) (entries 2 and 3). ${ }^{15}$ 
p-Methoxyacetophenone 7 was converted to the corresponding $(R)$-alcohol 14 with good enantioselectivity (89\%) and chemical yield (91\%) (entry 4). ${ }^{16}$ Compound 8 with a methyl substituent at the 4-position on the aryl ring provided the $R$-alcohol 15 in high enantioselectivity $(90 \%)$ and very good yield (92\%) (entry 5). Finally, the asymmetric reduction of the cyclic aryl ketones 9 and 10 under the same conditions gave excellent chemical yields (up to 97\%) and even better enantioselectivities (Table 1). Reduction of 1-indanone 9 proceeded smoothly to give $(R)$ 2,3-dihydro- $1 H$-inden-1-ol 16 in high enantioselectivity (ee $95 \%$, entry 6$)^{16}$ and reduction of $\alpha$ tetralone 10 produced the corresponding $(R)$-alcohol 17 with the best enantioselectivity $(96 \%)$ (entry 7). ${ }^{17}$

In order to determine the minimum amount of reagent required to obtain the maximum selectivity, the reduction of $\alpha$-tetralone $\mathbf{1 0}$ was performed with varying amounts $(2,5,7$ and 10 mol \%) of chiral 2-methyl-1,3,2-dioxaborolane catalyst 3 (Table 2).

The results show that there is only marginal dependence of the ee from the amount of chiral catalyst. In addition, we also studied the effect of reaction temperature on ee and chemical yield (Table 3).

Table 2. Asymmetric reduction of $\alpha$-tetralone $\mathbf{1 0}$ with varying amounts of chiral auxiliary $\mathbf{3}$

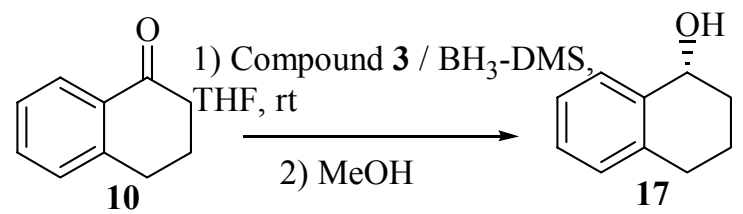

\begin{tabular}{cccc}
\hline Entry & Compound $\mathbf{3}(\mathrm{mol} \%)$ & Yield $^{\mathrm{a}} \mathbf{( \% )} \mathbf{1 7}$ & Enantiomeric purity $^{\mathrm{b}}(\mathbf{\%}) \mathbf{1 7}$ \\
\hline 1 & 2 & 98 & 90 \\
2 & 5 & 97 & 90 \\
3 & 7 & 97 & 92 \\
4 & 10 & 98 & 96 \\
\hline
\end{tabular}

${ }^{a}$ Chemical yields of isolated product. ${ }^{b}$ Enantiomeric excesses determined by chiral HPLC using the Chiralcel OD-H column.

Table 3. Effect of temperature on enantioselectivity of reduction of $\alpha$-tetralone $\mathbf{1 0}$ using $\mathbf{3}$ (10 mol \%)

\begin{tabular}{cccc}
\hline Entry & Temperature $\left({ }^{\circ} \mathrm{C}\right)$ & Yield $^{\mathrm{a}}(\%) \mathbf{1 7}$ & Enantiomeric purity $^{\mathrm{b}}(\%) \mathbf{1 7}$ \\
\hline 1 & -78 & 45 & 50 \\
2 & -10 to 0 & 60 & 77 \\
3 & rt $(25)$ & 98 & 96 \\
4 & 50 & 20 & 88 \\
\hline
\end{tabular}

${ }^{a}$ Chemical yields of isolated product. ${ }^{b}$ Enantiomeric excesses were determinate by chiral HPLC using the chiral column, Chiralcel OD-H. 
Interestingly, room temperature seems optimal for the asymmetric reductions using compound 3 as the borane-coordinating auxiliary. The auxiliary may not be stable at $50{ }^{\circ} \mathrm{C}$ and at $-78^{\circ} \mathrm{C}$ the auxiliary formation may be too slow. ${ }^{18}$

\section{Conclusions}

The asymmetric reduction of prochiral ketones 4-10 using a new chiral auxiliary, 2-methoxy1,3,2-dioxaborolane 3, in the borane reduction was studied. The catalyst was derived from $2 \alpha, 3 \alpha$-dihydroxycativic acid, a natural product isolated from the aerial parts of Baccharis scandens DC. Steric and/or electronic factors were discussed. In the cyclic aryl ketones series, compound 3 reduced 1-indanone 9 and $\alpha$-tetralone $\mathbf{1 0}$ in excellent ee (95 and 96\% ee, respectively). Aryl methyl ketones derived from acetophenone showed good chemical yields and moderated to good enantioselectivities. In addition, the effect of temperature and amount of chiral reagent on the enantioselectivity of the reduction were also reported.

\section{Experimental Section}

General. Ketones 1-8, borane-dimethylsulfide and trimethoxyborane were obtained from Aldrich Chemical Co. All reactions were carried out under a dry nitrogen atmosphere. The purity of all reagents was checked by NMR spectroscopy. Dry toluene was used. THF was freshly distilled over sodium before use. CC was performed on silica gel G 70-230 mesh. ${ }^{1} \mathrm{H}$ NMR spectra were recorded in $\mathrm{CDCl}_{3}$ at $200.13 \mathrm{MHz}$, whereas ${ }^{13} \mathrm{C}$ NMR spectra were obtained at $50.23 \mathrm{MHz}$ on a Bruker AC-200 using TMS as an internal standard. Optical rotations were obtained on a Perkin-Elmer 341 polarimeter, and IR spectra were recorded on a Bruker IFS-2S spectrometer. Enantiomeric excesses were determined by HPLC with Daicel Chiralcel OD-H column or by CG with Supelco $\beta$-DEX 120 column.

Isolation procedure. From aerial parts of $B$. scandens DC., was isolated $2 \alpha, 3 \alpha$-dihydroxycativic acid (1) (45 mg/500 g plant) using CC on silica gel G 70-230 mesh eluting with $n$-hexane, $n$ hexane/EtOAc with increasing polarity mixtures and $\mathrm{EtOAc} / \mathrm{MeOH}$ (97:3).

Synthesis of 2-methoxy-1,3,2-dioxaborolane (3). Methylation with diazomethane and Pdcatalyzed hydrogenation of $\mathbf{1}$ produced $\mathbf{2}$. To stirred mixture of compound $2(17.7 \mathrm{mg}, 0.05$ $\mathrm{mmol})$ in dry toluene $(1 \mathrm{~mL})$ in a three necked flask was added trimethoxyborane $\left[\mathrm{B}(\mathrm{OMe})_{3}\right]$ $(0.01 \mathrm{~mL}, 0.1 \mathrm{mmol})$ at room temperature under an nitrogen atmosphere. The reaction mixture was stirred and heated under reflux for $8 \mathrm{~h}$. The reaction was monitored by TLC. After compound 3 was consumed, the excess $\mathrm{B}(\mathrm{OMe})_{3}$ was evaporated off. Yield $98 \%$. Amorphous solid; $[\alpha]_{D}^{25}=-39.9\left(\mathrm{c} 0.77, \mathrm{CHCl}_{3}\right) ; \mathrm{IR}\left(v_{\max }, \mathrm{cm}^{-1}\right)=1978,1644,1389,1360,1323,1165 .{ }^{1} \mathrm{H}$ NMR (300 MHz, CDCl $) \delta 0.79(3 \mathrm{H}, \mathrm{s}, \mathrm{C}-20-\mathrm{Me}), 0.89(3 \mathrm{H}, \mathrm{s}, \mathrm{C}-18-\mathrm{Me}), 0.92(3 \mathrm{H}, \mathrm{d}, J=7 \mathrm{~Hz}$, 
C-17-Me), 0.97 (3H, d, $J=7.1 \mathrm{~Hz}, \mathrm{C}-16-\mathrm{Me}), 0.99$ (3H, s, C-19-Me), 1.55 (1H, m, C-1 $\alpha-\mathrm{H})$, $1.55(1 \mathrm{H}, \mathrm{m}, \mathrm{C}-5-\mathrm{H}), 1.86(1 \mathrm{H}, \mathrm{m}, \mathrm{C}-1 \beta-\mathrm{H}), 2.28(2 \mathrm{H}, \mathrm{d}, J=7.3 \mathrm{~Hz}, \mathrm{C}-14-\mathrm{H}), 3,50$ (1H, brs, C3-H), 3.65 (3H, s, $\left.-\mathrm{CO}_{2} \mathrm{Me}\right), 3.75$ (3H, s, -OMe), 4.12 (1H, ddd, $\left.J=10.3,6.0,4.4 \mathrm{~Hz}, \mathrm{C}-2-\mathrm{H}\right)$. ${ }^{13} \mathrm{C}$ NMR $\left(75 \mathrm{MHz}, \mathrm{CDCl}_{3}\right) \delta 14.3 \mathrm{q}(\mathrm{C}-20), 20.0 \mathrm{q}(\mathrm{C}-16), 21.8 \mathrm{q}(\mathrm{C}-18), 21.8 \mathrm{q}(\mathrm{C}-17), 23.1 \mathrm{t}$ (C-6), 23.3 t (C-7), 24.9 t (C-11), 28.0 q (C-19), $31.1 \mathrm{~d}(\mathrm{C}-13), 37.8 \mathrm{~s}(\mathrm{C}-10)^{*}, 38.1 \mathrm{~s}(\mathrm{C}-4)^{*}, 39.2$ $\mathrm{t}(\mathrm{C}-12), 39.6 \mathrm{t}(\mathrm{C}-1), 41.3 \mathrm{t}$ (41.3), $42.8 \mathrm{~d}(\mathrm{C}-5), 51.3 \mathrm{q}(\mathrm{OMe}), 51.9 \mathrm{q}(\mathrm{OMe}), 54.6 \mathrm{~d}(\mathrm{C}-8), 54.8$ $\mathrm{d}(\mathrm{C}-9), 67,7 \mathrm{~d}(\mathrm{C}-2), 79.0 \mathrm{~d}(\mathrm{C}-3), 171.8 \mathrm{q}(\mathrm{C}=\mathrm{O}),{ }^{*}$ (exchanges).

\section{Typical procedure for the asymmetric reduction of ketones (1-8)}

To a THF $(1 \mathrm{~mL})$ solution of dioxaborolane $3(0.132 \mathrm{mg}, 0.10 \mathrm{mmol})$ prepared above was added $2 \mathrm{M} \mathrm{BH}_{3} \cdot \mathrm{Me}_{2} \mathrm{~S}(28 \mu \mathrm{L}, 0.30 \mathrm{mmol})$. After being stirred at room temperature for $10 \mathrm{~min}$, the solution of acetophenone $4(120 \mu \mathrm{L}, 1 \mathrm{mmol})$ in THF $(2 \mathrm{~mL})$ was added via syringe pump over 1 $\mathrm{h}$. The reaction mixture was stirred for $5 \mathrm{~h}$ at room temperature. The reaction mixture was quenched with $\mathrm{MeOH}(1 \mathrm{~mL})$. Solvent was removed under reduced pressure. The residue was flash chromatographed though a small pad of silica gel using $n$-hexane/ethyl acetate $(9: 1)$ as solvent.

(R)-1-Phenylethanol (11). Colorless oil in 93\% yield; ${ }^{1} \mathrm{H}$ NMR $\left(\mathrm{CDCl}_{3}\right) \delta 1.44(3 \mathrm{H}, \mathrm{d}, J=6.52$ $\mathrm{Hz}), 3.89(1 \mathrm{H}, \mathrm{s}), 4.52-4.85(1 \mathrm{H}, \mathrm{m}), 7.26-7.38(5 \mathrm{H}, \mathrm{m})$. Enantiomer determination of 11: 64\% $e e$ based on chiral HPLC [Daicel Chiralcel OD-H column, $0.4 \mathrm{~mL} / \mathrm{min}, 254 \mathrm{~nm}, n$-hexane $/ i$ $\mathrm{PrOH}$ (95:5), $t_{1}$ (R-isomer): $16.6 \mathrm{~min}, t_{2}(S$-isomer): $20.3 \mathrm{~min}] .[\alpha]_{\mathrm{D}}^{25}=+24.9$ (c 0.77 , $\left.\mathrm{CHCl}_{3}\right)^{17,19}$

(R)-1-(4-Chlorophenyl)ethanol (12). Colorless oil in 89\% yield. ${ }^{1} \mathrm{H}$ NMR $\left(\mathrm{CDCl}_{3}\right) \delta 1.43(3 \mathrm{H}$, d, $J=6.34 \mathrm{~Hz}), 2.34(1 \mathrm{H}, \mathrm{s}), 4.82(1 \mathrm{H}, \mathrm{q}, J=6.34 \mathrm{~Hz}), 7.25-7.30(4 \mathrm{H}, \mathrm{m})$. Enantiomer determination of 12: 91\% ee based on chiral HPLC [Daicel Chiralcel OD-H, $0.4 \mathrm{~mL} / \mathrm{min}, 254$ nm, $n$-hexane/ $i$-PrOH, $(95: 5), t_{1}\left(R\right.$-isomer): $30.0 \mathrm{~min}, t_{2}(\mathrm{~S}$-isomer $\left.): 28.9 \mathrm{~min}\right] .[\alpha]_{\mathrm{D}}^{25}=+20.5(\mathrm{c}$ $\left.0.74, \mathrm{CHCl}_{3}\right){ }^{19}$

(R)-1-(4-Bromophenyl)ethanol (13). Colorless oil in 94\% yield. ${ }^{1} \mathrm{H}$ NMR $\left(\mathrm{CDCl}_{3}\right) \delta 1.44(3 \mathrm{H}$, $\mathrm{d}, J=6.28 \mathrm{~Hz}), 2.35(1 \mathrm{H}, \mathrm{s}), 4.80(1 \mathrm{H}, \mathrm{q}, J=6.28 \mathrm{~Hz}), 7.45-7.50(4 \mathrm{H}, \mathrm{m})$. Enantiomer determination of 13: $93 \%$ ee based on chiral HPLC [Daicel Chiralcel OD-H column, 0.4 $\mathrm{mL} / \mathrm{min}, 254 \mathrm{~nm}, n$-hexane $/ i-\mathrm{PrOH},(95: 5), t_{1}(R$-isomer $)=31.0 \mathrm{~min}, t_{2}(S$-isomer $\left.)=29.1 \mathrm{~min}\right]$. $[\alpha]_{\mathrm{D}}^{25}=+27.1\left(\mathrm{c} 0.85, \mathrm{CHCl}_{3}\right) .{ }^{19}$

(R)-1-(4-Methoxyphenyl)ethanol (14). Colorless oil in 91\% yield. ${ }^{1} \mathrm{H}$ NMR $\left(\mathrm{CDCl}_{3}\right) \delta 1.49$ $(3 \mathrm{H}, \mathrm{d}, J=6.30 \mathrm{~Hz}), 1.83(1 \mathrm{H}, \mathrm{s}), 3.80(3 \mathrm{H}, \mathrm{s}), 4.86(1 \mathrm{H}, \mathrm{s}), 6.88-7.31(4 \mathrm{H}, \mathrm{m})$. Enantiomer determination of 14: $89 \%$ ee based on chiral GC [Supelco $\beta$-DEX 120 column, $40-180{ }^{\circ} \mathrm{C}$, programming rate $=2.0, t_{1}(\mathrm{R}$-isomer $)=66.2 \mathrm{~min}, t_{2}(\mathrm{~S}$-isomer $\left.)=67.0 \mathrm{~min}\right]$; HPLC (Daicel Chiralcel OD-H column, $0.3 \mathrm{~mL} / \mathrm{min}, n$-hexane $/ i$-PrOH $(95: 5), t_{1}(R$-isomer $)=36.8 \mathrm{~min}, t_{2}(S$ isomer $)=41.8 \mathrm{~min}) .[\alpha]_{\mathrm{D}}^{25}=+39.4\left(\mathrm{c} 0.82, \mathrm{CHCl}_{3}\right) .{ }^{17}$

(R)-1-(4-Methylphenyl)ethanol (15). Colorless oil in 92\% yield. ${ }^{1} \mathrm{H}$ NMR $\left(\mathrm{CDCl}_{3}\right) \delta 1.34(3 \mathrm{H}$, $\mathrm{d}, J=6.34 \mathrm{~Hz}), 2.23(3 \mathrm{H}, \mathrm{s}), 2.39(1 \mathrm{H}, \mathrm{s}), 4.69(1 \mathrm{H}, \mathrm{q}, J=6.34 \mathrm{~Hz}), 7.03(2 \mathrm{H}, \mathrm{d}, J=7.81 \mathrm{~Hz})$, $7.12(2 \mathrm{H}, \mathrm{d}, J=7.81 \mathrm{~Hz})$. Enantiomer determination of 15: 90\% ee based on chiral GC [Supelco 
$\beta$-DEX 120 column, $40-180{ }^{\circ} \mathrm{C}$, programming rate $=2.0, t_{1}(R$-isomer $)=54.7 \mathrm{~min}, t_{2}(S$-isomer $)$ $=56.2 \mathrm{~min}] .[\alpha]_{\mathrm{D}}^{25}=+31.4\left(\mathrm{c} 0.79, \mathrm{CHCl}_{3}\right){ }^{19}$

(R)-1-Indanol (16). Colorless oil in 98\% yield. ${ }^{1} \mathrm{H}$ NMR $\left(\mathrm{CDCl}_{3}\right) \delta 0.41(\mathrm{~m}, 1 \mathrm{H}, \mathrm{C} 8-\mathrm{H}), 1.87$ $(1 \mathrm{H}, \mathrm{m}, \mathrm{C} 8-\mathrm{H}), 2.75(1 \mathrm{H}, \mathrm{m}, 1 \mathrm{H}), 2.99$ (1H, m, C9-H), 5.16 (1H, t, J=6.2 Hz, C1-H); 7.15-7.19 $(3 \mathrm{H}, \mathrm{m}, \mathrm{Ar}), 7.35(1 \mathrm{H}, \mathrm{d}, J=5.6 \mathrm{~Hz}, \mathrm{Ar})$. Enantiomer determination of 16: $95 \%$ ee based on chiral GC [Supelco $\beta$-DEX 120 column, $40-180{ }^{\circ} \mathrm{C}$, programming rate $=2.0, t_{1}(R$-isomer $)=$ $66.2 \mathrm{~min}, t_{2}(S$-isomer $)=67.0 \mathrm{~min}$ ]; HPLC [Daicel Chiralcel OD-H column, $0.3 \mathrm{~mL} / \mathrm{min}, n$ hexane $/ i$-PrOH $(95: 5), t_{1}(R$-isomer $)=36.8 \mathrm{~min}, t_{2}(S$-isomer $\left.)=41.8 \mathrm{~min}\right] .[\alpha]_{\mathrm{D}}^{25}=-79.5(\mathrm{c} 0.09$, $\left.\mathrm{CHCl}_{3}\right){ }^{16}$

(R)-1,2,3,4-Tetrahydronaphthalen-1-ol (17). Colorless oil in 98\% yield. ${ }^{1} \mathrm{H} \mathrm{NMR}\left(\mathrm{CDCl}_{3}\right) \delta$ $1.64(1 \mathrm{H}, \mathrm{m}, \mathrm{C} 10-\mathrm{H}), 1.87(1 \mathrm{H}, \mathrm{m}, \mathrm{C} 10-\mathrm{H}), 2.20(1 \mathrm{H}, \mathrm{m}, \mathrm{C} 9-\mathrm{H}), 2.75(1 \mathrm{H}, \mathrm{m}, \mathrm{C} 9-\mathrm{H}), 2.85(1 \mathrm{H}$, m, C-8-H), 2.99 (1H, m, C8-H), $4.96(1 \mathrm{H}, \mathrm{t}, J=6.2 \mathrm{~Hz}, \mathrm{C} 1-\mathrm{H}), 7.10-7.22(3 \mathrm{H}, \mathrm{m}, \mathrm{Ar}), 7.38(1 \mathrm{H}$, $\mathrm{d}, J=5.6 \mathrm{~Hz}, \mathrm{Ar}$ ). Enantiomer determination of 17: $96 \%$ ee based on chiral GC [Supelco $\beta$ DEX 120 column, $80{ }^{\circ} \mathrm{C}, 10 \mathrm{~min} ; 5^{\circ} \mathrm{C} / \mathrm{min}$ to $110{ }^{\circ} \mathrm{C}, 8 \mathrm{~min}$; and $15{ }^{\circ} \mathrm{C} / \mathrm{min}$ to $160{ }^{\circ} \mathrm{C}, 11 \mathrm{~min}$. Enantiomer retention times: $t_{1}(R$-isomer $)=28.3 \mathrm{~min}, t_{2}(S$-isomer $\left.)=28.7 \mathrm{~min}\right] .[\alpha]^{25}{ }_{\mathrm{D}}=-20.1(\mathrm{c}$ $\left.0.75, \mathrm{CHCl}_{3}\right){ }^{19}$

\section{Acknowledgements}

Financial support was provided by CONICET (PIP 00628), ANPCyT (PICT 2007-352) and UNSL (22-Q508/7301).

\section{References}

1. (a) Silverman, R. B. The Organic Chemistry of Drug Design and Drug Action; Elsevier Academic Press: Burlington, MA, 2004, pp 7-105. (b) Challenger, C. A. Chiral Drugs and Chiral Intermediates; John Wiley \& Sons: New York, 2001, Vol. 3.

2. Morris, D. J.; Hayes, A. M.; Wills, M. J. Org. Chem. 2006, 71, 7035.

3. Schiffers, I.; Rantanen, T.; Schmidt, F.; Bergmans, W.; Zani, L.; Bolm, C. J. Org. Chem. 2006, 71, 2320.

4. (a) Kurbanoglu, E. B.; Zilbeyaz, K.; Kurbanoglu, N. I.; Kilic, H. Tetrahedron: Asymmetry 2007, 18, 2332. (b) Borén, L.; Martin-Matute, B.; Xu, Y.; Cordova, A.; Bäckvall, J. E. Chem. Eur. J. 2006, 12, 225. (c) Zhu, D.; Hua, L. J. Org. Chem. 2006, 71, 9484.

5. Fang, T.; Xu, J.; Du, D.-M. Synlett 2006, 1559.

6. (a) Brown, H. C.; Zaidlewicz, M. Recent Developments, Organic Syntheses via Boranes; Aldrich Chemical Company: Milwaukee, 2001, Vol. 2 (b) Matteson, D. S. Stereodirected Synthesis with Organoboranes; Springer: Berlin, Heidelberg, 1995. 
7. (a) Balakrishnan, U.; Ananthi, N.; Velmath, S. Tetrahedron: Asymmetry 2009, 10, 1150. (b) Matsuo, J. -I.; Kozai, T.; Nishikawa, O.; Hattori, Y.; Ishibashi, H. J. Org. Chem. 2008, 73, 6902. (c) Lohray, B. B.; Bhushan, V. Angew. Chem., Int. Ed. 1992, 31, 729.

8. Matteson, D. S. Organoboranes for Syntheses; ACS Symposium Series, American Chemical Society: Washington, DC, 2001; Vol. 783, p 207.

9. Štefane, B.; Polanc, S. Synlett 2004, 698.

10. Krzemiński, M. P.; Wojtczak, A. Tetrahedron Lett. 2005, 46, 8299.

11. (a) Brown, H. C.; Ramachandran, P. V. Acc. Chem. Res. 1992, 25, 16. (b) Ramachandran, P. V.; Krzemiński, M. P.; Ram Reddy, M. V.; Brown, H. C. Tetrahedron: Asymmetry 1999, 10, 11. (c) Matteson, D. S.; Man, H.-W.; Ho, O. C. J. Am. Chem. Soc. 1996, 118, 4560. (d) Malhotra, S. V. Tetrahedron: Asymmetry 2003, 14, 645.

12. Gianello, J. C.; Pestchanker, M. J.; Tonn, C. E.; Guo, M.; Giordano, O. S. Phytochem. 1990, 29, 656.

13. Salazar-Pereda, V.; Martinez Martinez, L.; Flores-Parra, A.; De Jesús Rosales-Hoz, M.; Ariza-Castoldo, A.; Contreras, R. Heteroat. Chem. 1994, 5, 139.

14. (a) Korenaga, T.; Kobayashi, F.; Nomura, K.; Nagao, S.; Sakai, T. J. Fluor. Chem. 2007, 128, 1153. (b) Corey, E. J.; Helal, C. J. Angew. Chem., Int. Ed. 1998, 37, 1987. (c) Quallich, G. J.; Blake, J. F.; Woodal, T. M. J. Am. Chem. Soc. 1994, 116, 8516.

15. Nakamura, K.; Matsuda, T. J. Org. Chem. 1998, 63, 8957.

16. Stepanenko, V.; De Jesús, M.; Correa, W.; Guzmán, I.; Vázquez, C.; De la Cruz, W.; OrtizMarcialesa, M.; Barnesb, C.L. Tetrahedron Lett. 2007, 48, 5799.

17. Palmer, M.; Walsgroue, T. J. Org. Chem. 1997, 62, 5226.

18. Santhi, V.; Rao J. M. Tetrahedron: Asymmetry 2000, 11, 3553.

19. Basavaiah, D.; Reddy G. J.; Rao, K. V. Tetrahedron: Asymmetry 2004, 15, 1881. 